\title{
Cosmic-ray isotope measurements with HELIX
}

\section{Nahee Park ${ }^{a, *}$ on behalf of the HELIX Collaboration}

(a complete list of authors can be found at the end of the proceedings)

${ }^{a}$ Queen's University, Department of Physics, Engineering Physics and Astronomy, 64 Bader Lane, Kingston, Canada

E-mail: nahee.park@queensu.ca

Recent discoveries of new features in Galactic cosmic-ray fluxes emphasize the importance of understanding the propagation of cosmic rays. HELIX (High Energy Light Isotope eXperiment) is designed to improve the measurements of light cosmic-ray isotopes, including the propagation clock isotope $10 \mathrm{Be}$ and stable secondary isotope $9 \mathrm{Be}$, which will be essential to study the propagation of the cosmic rays. The magnetic spectrometer of HELIX consists of a 1 Tesla superconducting magnet containing a high-resolution gas drift chamber as a tracking detector and two velocity measuring detectors: a time-of-flight detector and a ring-imaging Cherenkov detector. While the HELIX instrument can measure the fluxes of the light isotopes from protons $(\mathrm{Z}=1)$ up to neon $(\mathrm{Z}=10)$, it is optimized to study the Be flux from $0.2 \mathrm{GeV} / \mathrm{n}$ to beyond $3 \mathrm{GeV} / \mathrm{n}$ with a sufficient mass resolution to discriminate between $10 \mathrm{Be}$ and $9 \mathrm{Be}$. In this talk, I will review the scientific goals and the design of the instrument and report its current status and project plans.

$37^{\text {th }}$ International Cosmic Ray Conference (ICRC 2021)

July 12 th - 23rd, 2021

Online - Berlin, Germany

\footnotetext{
${ }^{*}$ Presenter
} 


\section{Introduction}

Recent precision measurements by space-borne detectors such as AMS-02 have revealed unexpected new cosmic-ray features. One of the most surprising results is the detection of an excess of positrons above $25 \mathrm{GeV}$, which is significantly higher than expected from simple propagation models [1,2]. Various theories have been suggested to modify the traditional propagation models to explain this new discovery, including additional astrophysical positron sources (e.g.[3]), secondary particles generated around the source regions (e.g.[4]), and dark matter annihilation (e.g.[5]). There is also growing evidence of spectral hardening at a rigidity of around $300 \mathrm{GV}$ for the primary particles (e.g. protons and helium) as well as for the secondary particles (e.g. lithium and boron). The measurements may indicate more complicated propagation processes for the cosmic rays than previously assumed. To understand the nature of these new features, a better understanding of cosmic-ray propagation is necessary.

The most commonly used data to study the propagation of cosmic rays are the measurements of primary-to-secondary nuclei ratios, such as the boron-to-carbon ratio. By comparing the flux of secondary nuclei generated by the inelastic collisions of primary nuclei with the propagation medium to the flux of primary nuclei originating from the source, the total material path length traversed by the primary cosmic rays during their containment time in our Galaxy can be estimated. Measurements of radioactive isotopes with known decay times close to the containment time of the cosmic rays, such as the ${ }^{10} \mathrm{Be}$ isotope, can provide key data to understand the propagation of the cosmic rays. The best existing measurements of the ${ }^{10} \mathrm{Be}$ to ${ }^{9} \mathrm{Be}$ ratio are provided by the ISOMAX experiment [6], which has relatively large statistical errors.

The High Energy Light Isotope eXperiment (HELIX) is a series of new long-duration balloon payloads designed to measure light isotopes from $0.2 \mathrm{GeV} / \mathrm{n}$ eventually up to $\sim 10 \mathrm{GeV} / \mathrm{n}$. The first phase of HELIX is designed to measure isotopes from proton $(Z=1)$ up to neon $(Z=10)$ in the energy range from $0.2 \mathrm{GeV} / \mathrm{n}$ up to $\sim 3 \mathrm{GeV} / \mathrm{n}$ with the detector configuration optimized to measure the ratio of the beryllium isotopes-the ratio between radioactive ${ }^{10} \mathrm{Be}$ to stable ${ }^{9} \mathrm{Be}$. HELIX is aiming to have its first long-duration balloon flight from the Esrange space center in Sweden in the summer of 2022.

\section{Instruments}

The HELIX instrument, shown in the left panel of Figure 1, is a magnet spectrometer designed to measure the light isotopes from proton up to neon $(Z=10)$. The instrument is optimized to measure the ${ }^{10} \mathrm{Be}$ to ${ }^{9} \mathrm{Be}$ ratio in the energy range between $0.2 \mathrm{GeV} / \mathrm{n}$ up to $\sim 3 \mathrm{GeV} / \mathrm{n}$ with a mass resolution equal or better than $\sim 3 \%$. To achieve the mass resolution, HELIX is designed to provide rigidity measurements with uncertainty of $\sim 2.5 \%$ and velocity measurements with uncertainty of $\sim 0.1 \%$. The rigidity measurements are obtained by accurately measuring the bent trajectories of incident charged particles with a multi-wire gas chamber under a near-uniform $1 \mathrm{~T}$ magnetic field. Accurate time-of-flight (TOF) measurements of the particles provide sufficiently accurate velocities of particles up to $1 \mathrm{GeV} / \mathrm{n}$, at which point an aerogel radiator for the ring imaging Cherenkov (RICH) detector can be used to measure the velocity. The expected measurements of the beryllium isotopes by HELIX for 14 days of flight are shown in the right panel of Figure 1, along with the 
previous measurements. The HELIX instrument can collect more than $1500{ }^{10} \mathrm{Be}$ nuclei above 2 $\mathrm{GeV} / \mathrm{n}$ with 14 days of exposure time, and provide precision measurements of the ratio for the first time.

All of the electronics and mechanics integrated in HELIX should perform within a strong magnetic field. This requirement influenced the overall design of the instrument, including the subdetectors, power, thermal, and mechanical support. As traditional photomultiplier tubes (PMTs) do not collect signal efficiently under a strong magnetic field, silicon photomultipliers (SiPMs) were selected as the photon detectors for the TOF and RICH systems. Because of the higher thermal noise in the SiPMs, careful thermal system design throughout the payload has been carried out that includes an active cooling system and intensive temperature monitoring. Electronic devices, especially those with inductors like DC/DC converters, were individually tested under appropriate magnetic fields, and were covered by magnetic field shielding if necessary. Because the strong magnetic field of HELIX causes the instrument to align with the geomagnetic field, an omnidirectional solar panel array was used instead of a directional solar panel design. Many mechanical structures were built with non-magnetic material, and the impact of the magnetic field was examined when ferromagnetic material had to be used.

A brief description of the detector and the current status of each subsystem is presented in the following sections.

\subsection{Time-of-Flight (TOF) and Charge System}

The HELIX TOF and charge detector is designed to measure the velocity and charge(Ze) of the incident nuclei and to provide the system-wide trigger. Three layers of $1 \mathrm{~cm}$ thick EJ-200 sincillator are located at the top, middle, and bottom of the detector. The top and bottom TOF layers each consists of eight scintillator paddles with $20 \mathrm{~cm}$ width and $1.6 \mathrm{~m}$ length, and are separated by $2.3 \mathrm{~m}$. Each end of the scintillator is read by eight SiPMs, mounted on two carrier boards (four
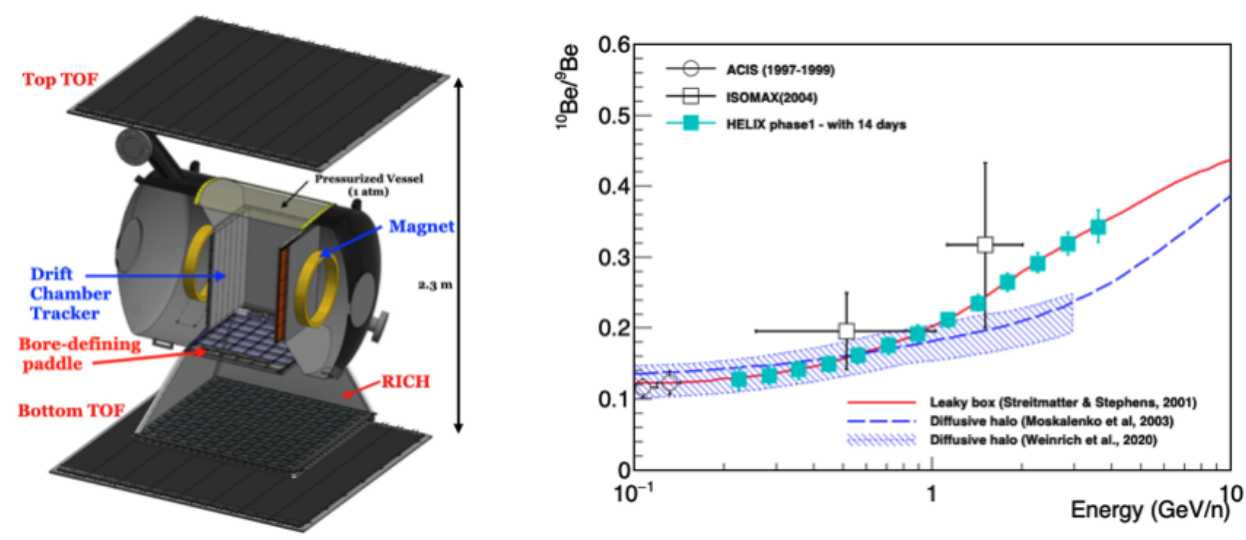

Figure 1: Left: Partially sectioned 3D model of the High Energy Light Isotope eXperiment detector systems, showing the superconducting magnet, DCT, two TOF layers, and RICH. Right: Expected beryllium isotope measurements with the HELIX instrument with 14 days of exposure time compared with previous measurements. The solid red line shows the expectation from a leaky box model [7], and the dashed blue line shows the expectation from a diffuse halo model [8]. The range of other diffuse halo models [9] is shown as a blue shaded area. 


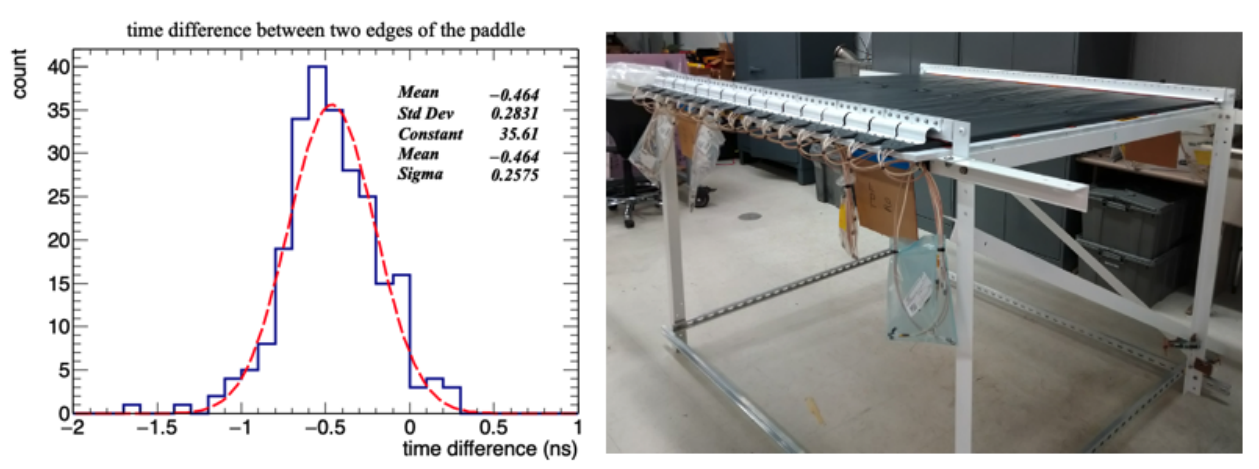

Figure 2: Left: Time difference between the two edges of a scintillator paddle as measured by the flight readout system. The peak is shifted because the triggering scintillator's location was slightly off from the center of the paddle. Right: Top TOF layer on the HELIX upper gondola frame.

SiPMs per board.) Hamamatsu S13360-6050VE SiPMs were selected for readout because of their dynamic range and high photo-detection efficiency, and low transit time skew. The middle layer, the bore-defining paddle, is designed to constrain the trigger geometry and to reduce the trigger rate by only accepting particles passing through the center of the detector. It is installed between the DCT and the radiator of the RICH. With dimensions of $0.6 \mathrm{~m}$ by $0.6 \mathrm{~m}$, it is smaller than the two main layers. Two opposite edges of the bore-defining paddle are each read out by $16 \mathrm{SiPMs}$, mounted on four carrier boards (four SiPMs per board.)

The SiPM carrier board handles the output of four SiPMs, and provides a fast signal output and a slow signal output to the readout board where the timing and charge information is processed. The output of each SiPM is divided into two-fast and slow signal paths, the fast signal being used for timing and the slow one for charge determination. The signal going to the fast path is connected to a fast amplifier that amplifies the signal by a factor of $\sim 18$. The four amplified signals from the SiPMs are combined and further amplified by an additional fast amplifier to form the fast signal output of the carrier board. All four signals of the slow path are combined and form the slow output of the carrier board. Each SiPM's operational bias voltage is set to achieve uniform gain. As the temperature-dependent gain changes are similar between SiPMs, the voltage is automatically adjusted for gain stabilization based on a common temperature sensor located on the SiPM carrier board.

Each TOF readout board handles signals from eight SiPM carrier boards. The amplified fast output from the SiPM carrier board is used to measure the timing of the signal at the readout board. The DC-coupled fast output is connected to a leading-edge discriminator. The leading edge of the output of the discriminator is connected to a Time-to-Amplitude Converter, whose output is sampled by a 14-bit ADC with a sampling speed of 40 MSPS. This TDC of the readout board has a timing resolution better than $25 \mathrm{psec}$. The slow output from the SiPM carrier board is used to measure the charge amplitude of the signal. After passing through a shaper, the slow signal is sampled with another 40 MSPS ADC channel. The charge information collected by the readout board can handle a signal up to 150 minimum ionizing particles (MIPs) signal with a resolution better than 0.1 MIP. The slow output is also used to provide an overall instrument trigger. Two discriminators with independent control signals provide two different trigger levels: one for a low-charge incident particle, and the other for a high-charge particle. 
The TOF system is designed to provide a timing resolution better than 50 pico-seconds(ps) for nuclei heavier than Lithium $(Z=3)$, which is sufficient to measure the velocity of these particles up to $1 \mathrm{GeV} / \mathrm{n}$ with good precision. A simple analysis of the initial test data with a muon trigger setup showed a timing resolution of $\sim 260 \mathrm{psec}$ as shown in the left panel of Figure 2, which agrees with expectations for MIP-level energy deposits. The three TOF layers measure the charge of incident particles up to $Z=10$ with charge resolution better than $0.1 \mathrm{e}$.

\subsection{Superconducting Magnet}

At the center of HELIX lies the superconducting magnet, which provides an approximately uniform $1 \mathrm{~T}$ magnetic field within a rectangular warm bore. Two superconducting coils inside the magnet are immersed in a liquid-helium (LHe) bath to keep the coil temperature lower than the critical temperature of $9.8 \mathrm{~K}$. The cryostat can hold 260 liters of LHe, providing a hold time of $\sim 7$ days. Initially designed and built for the HEAT experiment, the magnet has been flown successfully for five times where it was operated in $1 \mathrm{~atm}$ pressure maintained by a pressure vessel. The magnet was refurbished to make it functional in vacuum. The control and housekeeping system, including the discharging system, are also redesigned to function in a flight condition. The housekeeping information, such as shield temperatures and LHe level, are sent to the main housekeeping hub to be recorded. The persistent switch, which controls the current flow to the superconducting magnet, can be controlled by a flight command.

The magnetic field in the bore volume has been measured in three dimensions, and the measured values agree with the theoretical model. The refurbished magnet was tested at the flight pressure condition in 2019.

\subsection{Drift Chamber Tracker (DCT)}

The DCT, located inside the bore of the magnet, is designed to measure the rigidity and trajectory through the instrument of incident particles. The chamber has a volume of $45 \mathrm{~cm}$ by $45 \mathrm{~cm}$ by $58 \mathrm{~cm}$, which is fixed by the size of the magnet bore, the frame thickness needed to hold the wire tension and provide the necessary high voltage standoff, and the space needed for front-end electronics and cable routing. The DCT is installed inside a hermetic vessel that fits in the bore of the magnet and maintains the gas pressure at 1 atmosphere during flight. A gas mixture of $80 \% \mathrm{CO}_{2}$ and $20 \%$ Argon provides low thermal diffusion and good operating stability. The interior geometry of the DCT consists of 3 sense wire planes and 4 cathode planes. The interior cathode planes are constructed of $250 \mu \mathrm{m}$ gold-plated beryllium wire on a $4 \mathrm{~mm}$ pitch, while the outer cathode planes are solid gold-plated copper. Each sense wire plane consists of $7220 \mu \mathrm{m}$ resistive sense wires interspersed with potential wires operating at a nominal voltage of $-3 \mathrm{kV}$. The nominal cathode voltage is $-10 \mathrm{kV}$. The drift region between each cathode and sense wire plane is completely surrounded by field shaping electrodes consisting of a set of $4 \mathrm{~mm}$ pitch field shaping strips, providing a uniform drift field of $1.3 \mathrm{kV} / \mathrm{cm}$ in the drift volume. Resistive sense wires with a nominal resistance of $1.8 \mathrm{k} \Omega$ are used to provide charge-division based tracking in the non-bending view, while drift-time measurements provide high resolution tracking in the bending view. Each sense wire end is terminated at a chamber-mounted front-end (FE) board assembly. Sense wire signals are amplified by the FE board assembly and sent to the sampling ADC boards located outside of the gas chamber via custom hermetic feedthroughs. Each FE board assembly handles signals 
from 24 sense wires. ADC boards are housed in an electronics rack located next to the pressure vessel. Each ADC board processes the signal from 48 wire ends, and provides a full waveform readout of a $10 \mu \mathrm{sec}$ drift time window following an event trigger with a sample rate of 80 MSPS at 12 bit resolution. To reduce data size, the ADC board provides the ability to zero-suppress portions of the waveform falling below a programmable signal threshold, reading out only regions of interest (RoIs) of the full waveform in which a programmable signal threshold has been exceeded. The RoI threshold, pre-sampling length, and length of the RoI are programmable parameters. The ADC board provides a test pulse output to the front end electronics, used for functional testing.

Because the drift velocity is temperature-dependent, it is important to maintain a uniform temperature throughout the DCT to the extent possible, and to monitor variations in temperature within the DCT. Consequently, the outside surfaces of the DCT, with the exception of the two sides on which the FE assemblies are installed, are covered by thin Kapton heaters, used to compensate for the local heating generated by the FE assemblies. In addition, there are 24 precision thermistors installed on the outside of the DCT to monitor the chamber temperature profile.

The DCT is designed to achieve a mean spatial resolution of $65 \mu \mathrm{m}$ for $Z>3$ particles. The fully assembled DCT installed in the pressure vessel was installed in the magnet bore during a magnet vacuum operation test in 2019. It held 1 atm pressure in flight-like pressure conditions. Currently, the full integration test of the DCT is under way using cosmic-ray muons. Figure 3 shows a typical muon track measured by the DCT in the drift plane view.

\subsection{RICH}

For nuclei with energies higher than $1 \mathrm{GeV} / \mathrm{n}$, the

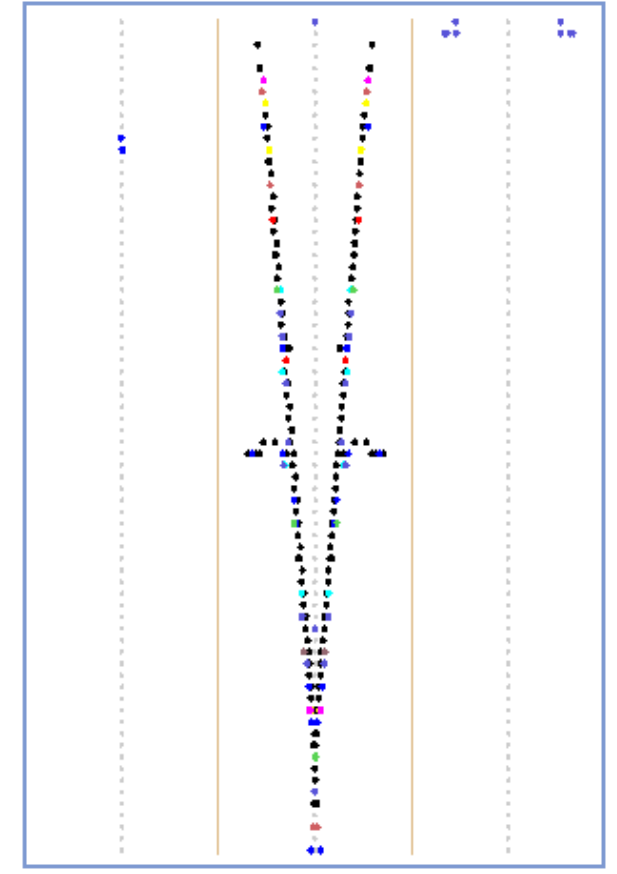

Figure 3: Event display for a muon event measured by the DCT before resolving the left-right ambiguity. Each colored dot shows the signal integrated over RoIs for the ADC channel. ring imaging Cherenkov detector ( $\mathrm{RICH})$ is used to estimate the velocity of the particles as the timing resolution of the TOF system is not sufficient to achieve the mass resolution needed to separate ${ }^{9} \mathrm{Be}$ from ${ }^{10} \mathrm{Be}$ at these energies. Located under the bore paddle, a proximity-focused RICH designed for HELIX consists of a $1 \mathrm{~cm}$ thickness radiator and a $1 \mathrm{~m}^{2}$ focal plane separated by an expansion length of $50 \mathrm{~cm}$. The radiator consists of a 6 by 6 array of radiator blocks, and each block has dimensions of $10 \mathrm{~cm}$ by $10 \mathrm{~cm}$. Transparent, hydrophobic aerogel tiles with a high refractive index of 1.16 fabricated by a pin-drying technique [10] are used as the main radiator. Four sodium fluoride blocks are installed as sub-radiator for the cross-calibration between TOF and RICH. Half of the focal plane is covered with 200 SiPM arrays in a checkboard pattern. Each SiPM array has 64 pixel sensors with a pixel size of $6 \times 6 \mathrm{~mm}^{2}$. To reduce the material in the particle's trajectories, the SiPM arrays are read by $70 \mathrm{~cm}$ long flexible PCB cables connecting to the readout boards mounted in electronic cages outside of the beam. To reduce the dark current, the focal plane 

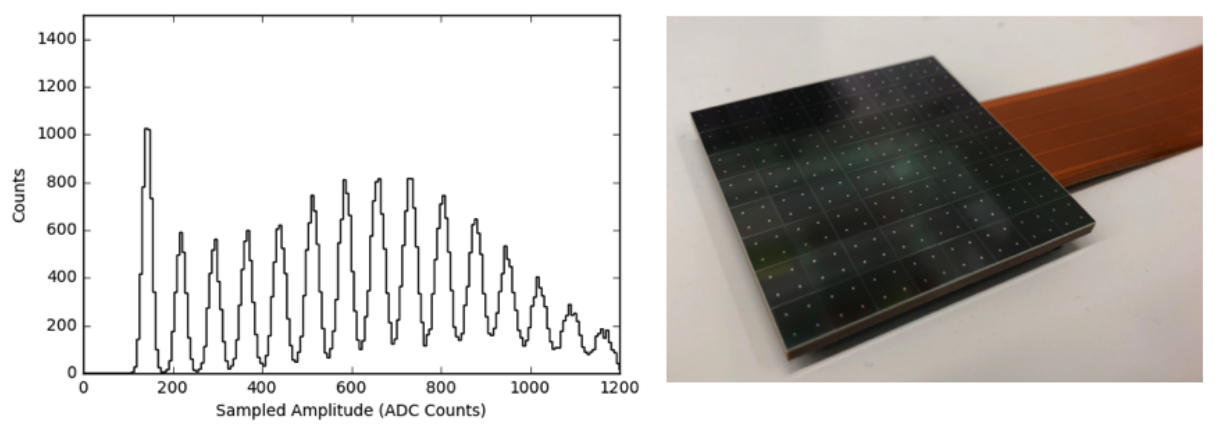

Figure 4: Left: Photoelectron spectrum read by the RICH readout board from a SiPM pixel. Right: SiPM array with flexible PCB.

is maintained at low temperature. The radiator and SiPM array is enclosed by a light-weight dark box structure.

The RICH readout board controls 16 CITIROC ASIC chips, with each chip reading output from 32 SiPM pixels. The output of a SiPM pixel read out by a CITIROC generates a fast trigger output and a charge output. The high gain preamplifier of the CITIROC is used for both trigger and charge output. The CITIROC provides an individual discriminator for each channel to generate a trigger output. The trigger output provides timing for each event with a resolution better than $12.5 \mathrm{nsec}$, which will be used to suppress random hits due to the thermal noise from the SiPMs. The charge output is determined by reading the pulse height captured by a sample-and-hold control signal. The CITIROC provides a multiplexed output of 32 channels' charge output, which is read by a 12-bit ADC in the readout board. The bias voltage, gain, and trigger threshold can be set for each individual channel. The readout board provides a trigger threshold as low as $0.3 \mathrm{pe}$. It is designed to support a charge output from a single pe up to 100 pe.

The RICH is designed to measure the velocity of nuclei with an uncertainty of $0.1 \%$ for nuclei with a charge higher than Lithium $(Z=3)$ and energy higher than $1 \mathrm{GeV} / \mathrm{n}$. To meet this design requirement, detailed calibration measurements were carried out to measure the properties of the aerogel blocks including the absolute refractive index better than $0.1 \%$ [11]. The performance of a RICH readout board was tested with a SiPM array by measuring a photo-spectrum with a light source, which showed clear pe peaks as shown in Figure 4. All the SiPM arrays and the readout boards are currently under characterization tests.

\section{Current status and plan}

While the pandemic has delayed the schedule significantly, steady progress has been made. The production of the flight hardware has finished, and its performance was tested. HELIX has started the instrument integration. The DCT and TOF systems will be integrated by July of 2021, ready for performance tests under a realistic magnetic field with a magnet cool down test. The full integration and performance tests will be held in the fall, aiming for a thermal-vacuum test for the payload in the upcoming winter season. We are currently planning to have a long-duration balloon flight from Sweden in summer 2022. 


\section{References}

[1] O. Adriani et al, 2009, Nature

[2] L. Accardo et al., 2014, PRL

[3] S. Manconi et al., 2020, PRD

[4] R. Yang \& F. Aharonian, 2019, PRD

[5] I. Cholis et al., 2009, PRD

[6] T. Hams et al., 2004, ApJ

[7] R.E. Streitmatter and S.A. Stephens, 2001, Adv. Space Res.

[8] I.V. Moskalenko et al., 2003, ApJ

[9] N. Weinrich et al., 2020, A\&A

[10] M. Tabata at al, 2019, RICH 2018 proceeding

[11] S. O'Brien at al., 2021, ICRC 


\section{Full Authors List: HELIX Collaboration}

P. Allison ${ }^{1}$, J. J. Beatty ${ }^{1}$, L. Beaufore ${ }^{2}$, Y. Chen ${ }^{3}$, S. Coutu ${ }^{3}$, M. Gebhard ${ }^{4}$, N. Green ${ }^{5}$, D. Hanna ${ }^{6}$, H. B. Jeon ${ }^{2}$, B. Kunkler ${ }^{4}$, M. Lang ${ }^{4}$, R. Mbarek ${ }^{2}$, K. McBride ${ }^{1}$, I. Mognet ${ }^{3}$, D. Müller ${ }^{2}$, J. Musser ${ }^{4}$, S. Nutter ${ }^{7}$, S. O’Brien ${ }^{6}$, N. Park $^{8}$, Z. Siegel ${ }^{2}$, M. Tabata ${ }^{9}$, G. Tarlé ${ }^{5}$, G. Visser ${ }^{4}$, S. P. Wakely ${ }^{2}$, M. $\mathrm{Yu}^{3}$

${ }^{1}$ The Ohio University, Department of Physics, 191 West Woodruff Ave, Columbus, USA. ${ }^{2}$ University of Chicago, Department of Physics, 5720 South Ellis Avenue, Chicago, USA. ${ }^{3}$ Pennsylvania State University, Department of Physics, University Park, USA. ${ }^{4}$ Indiana University, Department of Physics, 727 E. third St., Bloomington, USA. ${ }^{5}$ University of Michigan, Department of Physics, 450 Church Street, Ann Arbor, USA. ${ }^{6}$ McGill University, Department of Physics, 3600 rue University, Montreal, Canada. ${ }^{7}$ Northern Kentucky University, Department of Physics, Geology and Engineering Technology, Highland Heights, USA. ${ }^{8}$ Queen's University, Department of Physics, Engineering Physics and Astronomy, 64 Bader Lane, Kingston, Canada. ${ }^{9}$ Chiba University, Department of Physics, Chiba, Japan. 